\title{
Strain Differences in Morphometrical Characteristics of Rat Kidneys
}

\author{
Nanae MURATA ${ }^{1)}$, Akira YABUKI ${ }^{2}$, Mitsuharu MATSUMOTO') ${ }^{2}$, Hayao NISHINAKAGAWA ${ }^{2)}$ and Shusaku SUZUKI ${ }^{2)}$ \\ ${ }^{1)}$ National Veterinary Assay Laboratory, Ministry of Agriculture, Forestry and Fisheries, 1-15-1 Tokura Kokubunji, Tokyo 185-8511 and \\ ${ }^{2)}$ Laboratry of Veterinary Anatomy, Faculty of Agriculture, Kagoshima University, 21-24 Korimoto 1, Kagoshima 890-0065, Japan
}

(Received 5 April 2006/Accepted 21 July 2006)

ABSTRACT. To clarify the strain differences in the morphology of the rat kidneys, we investigated the morphometrical characteristics of the kidneys of Slc:Wistar, Slc:SD, and F344/NSlc rats. The diameter of the renal corpuscles in female F344/N rats is smaller than that in female Wistar rats. Although sex differences (males $>$ females) were shown in SD and F344/ $\mathrm{N}$ rats, no effects of castration were detected in any of the groups. Strain-dependent differences in the percentage of renal corpuscles with a cuboidal parietal layer were found in both male and female groups. The highest percentage of them was noted in male Wistar rats. Effects of castration were observed in female Wistar and male F344/N rats, and the values after castration were significantly higher than those in the intact animals. As for the number of proximal convoluted tubular nuclei, no strain differences were detected in either the male or female groups. Although a sex difference was found in SD rats (female>male), no effects of castration were detected in any of the groups. In female F344/N rats, numerous numbers of PAS-positive granules, which were observed in the proximal convoluted and straight tubular epithelia, were noted. Orchiectomy induced an increase of these granules in male SD and F344/N rats, but ovariectomy showed no effects on them in any strains. This is the first study to clarify the strain differences in the morphological characteristics of the kidneys in ordinary rat strains.

KEY WORDS: castration, kidney, rat, sex difference, strain difference.

J. Vet. Med. Sci. 68(11): 1185-1189, 2006

Rats are frequently used in animal experiments, and there have been many reports regarding the sex differences in the morphology and function of the rat kidneys; e.g., the segmentation of nephrons [13], the thickness of each of the renal zones [16], the ultrastructural features of the proximal tubular epithelium $[21,27]$, the concentration and constituents of the urine $[1,11,16]$, and the structural and functional alterations with aging $[3,6,18,22]$. The effects of castration and sex hormone-treatment in the rat kidneys have also been investigated, and the effects on the size of the glomeruli and proximal tubules [14], ultrastructural features of the proximal tubular epithelium [21, 27], concentration and constituents of urine $[11,16]$, and the severity of glomerular damage with aging or after uninephrectomy $[3,5,15,19$, 20], have been reported. Regarding the strain differences, several reports demonstrated the strain differences in the urinary constituents [1], glomerular filtration rate (GFR) [7], renal plasma flow (RPF) [7], pathological changes with aging $[6,18,22]$, and the sensitivity to nephrotoxicity due to chemical drugs [4, 17] and high-protein food [12].

Recently, we investigated the renal morphology in five mouse strains (ICR, BALB/cA, C3H/HeN, C57BL/6Cr and $\mathrm{DBA} / 2 \mathrm{Cr}$ ), and identified the sex and strain morphological differences; the percentage of the renal corpuscles exhibiting a cuboidal parietal layer (CPL), the diameter of the renal corpuscles, the number of nuclei in the proximal convoluted tubules (PCTs), the number of periodic acid Schiff (PAS)positive granules in the proximal straight tubules (PSTs), and the appearance of vacuolar structures in the PCTs [23, 24]. However, in the rat, strain differences in the morphological characteristic of the kidney have remained unclear. Moreover, we do not have enough knowledge as to whether sex differences in the rat renal morphology are dependent on the strains or not.

Therefore, in the present study, we morphometrically analyzed the kidneys sections prepared from male and female Wistar, SD, and F344/N rats, which are the major ordinary rat strains.

\section{MATERIALS AND METHODS}

Animals: Slc:Wistar, Slc:SD, and F344/NSlc rats were used for the experiment. Males and females from each strain were divided into intact ( $\mathrm{n}=4$ in each group) and castration groups ( $n=3$ in each group). All rats were housed in an open system room with a one-way airflow system (temperature $22 \pm 1^{\circ} \mathrm{C}$; humidity $55 \pm 10 \%$; light period $07: 00$ to 19:00; ventilation 12 cycles/hr) at the Frontier Science Research Center, Kagoshima University, and were given an autoclaved commercial diet (CE-2; Japan CLEA, Inc., Tokyo, Japan) and tap water ad libitum.

The present study was performed in accordance with the Guidelines for Animal Experimentation of Kagoshima University, Japan. All orchiectomies and ovariectomies were performed at one-month of age under anesthesia, using a mixture of ketamine and medetomidine. All rats were sacrificed at three-month of age by exsanguination from the carotid arteries under deep anesthesia after measuring their body weights, using pentobarbital. The kidneys were quickly removed.

Tissue preparation: Five-mm-thick central slices from the left kidneys, including the hilum, were cut perpendicular to the $\log$ axis and fixed in $10 \%$ neutral buffered formalin at room temperature. After routine embedding in paraffin, 3 
$\mu \mathrm{m}$-thick sections were selected every $30 \mu \mathrm{m}$ and stained with PAS.

Morphometric analyses: Five sections from each animal were used for the randomized morphometric analyses as follows: 1) Diameter of the renal corpuscles in the cortex: five cortical renal corpuscles with the vascular pole or the urinary pole were selected in each section (25 renal corpuscles/ each animal). The line connecting the vascular pole to the urinary pole was taken as the vertical axis, and the widest distance along the cross axis of the glomerular capsule at a right angle to the vertical axis was regarded as the diameter of the renal corpuscle [23]. 2) Percentage of the renal corpuscles with a CPL: the number of renal corpuscles with a CPL (A) and the total number of renal corpuscles (B) were counted in each section. The ratio of $\mathrm{A}$ to $\mathrm{B}$ was expressed as a percentage. 3) The number of nuclei of the PCTs: the number of PCT nuclei per unit area of cortex $\left(100,000 \mu \mathrm{m}^{2}\right)$ as a parameter of the PCTs size was counted in each section. 4) Number of PAS-positive granules in the PCTs: light micrographs of the cortex in each section were taken at a final magnification of 1,000, and the area of the PCTs epithelium, which excluded the area of the brush border, was measured using NIH image 1.62. The granules within this area were counted and expressed per $10,000 \mu \mathrm{m}^{2}$. 5) Number of PAS-positive granules in the PSTs: The same measurement technique was used in the area of outer cortex.

Statistical analyses: All data were expressed as the mean \pm standard error (S.E.). The significant differences between the two groups (differences between males and females, and differences between the intact and castrated groups) were analyzed using the Mann-Whitney $U$ test. The significant differences between multigroups (strain differences in each sex group) were analyzed using a one-way analysis of variance (ANOVA, Bonferroni/Dunn test). Statistical significance was defined as $P<0.05$. The statistical analyses were performed using Statview-J4.02 version on a Macintosh computer.

\section{RESULTS}

Strain- and sex differences in the intact rats: The diameter of the renal corpuscles in the cortex area showed strain differences in the female groups, and Wistar rats exhibited significantly higher values than F344/N rats. Sex differences were observed in SD and F344/N rats, and males exhibited significantly higher values than females in both strains (Fig. 1A).

Although the parietal layer of most of the renal corpuscles consisted of squamous epithelium, renal corpuscles with cuboidal epithelium cells in the parietal layer were occasionally observed (Fig. 2). In the percentage of renal corpuscles with a CPL, strain differences were observed in both male and female groups. Wistar rats exhibited significantly higher percentages of them than SD and F344/N rats in both sexes. Sex differences were observed in Wistar and SD rats, and males exhibited significantly higher percentages of them than females in both strains (Fig. 1B).
As for the number of PCT nuclei, no significant strain differences were observed in either of the sex groups. A sex difference was observed in SD rats, and females exhibited higher values than the males (Fig. 1C).

The PAS-positive granules were observed in the proximal tubular epithelium, and were more numerous in PCTs than in PSTs (Fig. 3). The number of granules in the PCTs showed significant strain differences in the female groups, and the number of them in F344/N rats was significantly higher than that in Wistar rats. Sex differences were observed in SD and F344/N rats, and the numbers of them in females were significantly higher than those in males in both strains (Fig. 1D). In the PSTs too, the number of PASpositive granules showed significant strain-differences in the female groups, and the number in female F344/N rats was significantly higher than that in Wistar rats. A sex difference was observed in F344/N rats, and the number of PAS-positive granules in females was significantly higher than that in males (Fig. 1E).

Effects of orchiectomy: In the diameter of the renal corpuscles in the cortex area, no significant changes after orchiectomy were observed in all strains (Fig. 1A). As for the percentage of the renal corpuscles with a CPL, orchiectomized F344/N rats exhibited significantly higher percentages of them than the intact male groups (Fig. 1B). The number of PCT nuclei showed no effects of orchiectomy in any of the strains (Fig. 1C). As for the number of PAS-positive granules in the PCTs, the orchiectomized SD rats exhibited significantly higher values than the intact males. (Fig. 1D). As for the number of PAS-positive granules in the PSTs, the orchiectomized SD and F344/N rats exhibited significantly higher values than the intact males.

Effects of ovariectomy: Regarding the diameter of the renal corpuscles in the cortex area, no significant changes after ovariectomy were observed in any of the strains (Fig. 1A). As for the percentage of the renal corpuscles with a CPL, ovariectomized Wistar rats exhibited significantly higher percentages than intact females (Fig. 1B). The number of PCT nuclei showed no effects of ovariectomy in any of the strains (Fig. 1C). Regarding the number of PAS-positive granules, no significant changes after ovariectomy were observed in any of the strains (Figs. 1D and 1E).

\section{DISCUSSION}

The renal corpuscle diameters from Wistar, SD, and F344/N rats were compared within each sex group, and significant strain differences were demonstrated only in the female group. Although sex differences were detected in $\mathrm{SD}$ and $\mathrm{F} 344 / \mathrm{N}$ rats, no effect of orchiectomy or ovariectomy was detected in either strain. In our previous report in mice, it was suggested that differences in the renal corpuscle diameter were closely correlated to the differences in the percentage of the renal corpuscles with a CPL [23]. However, in the present study, a close correlation between these two parameters was not observed in the rat kidneys. Therefore, it was suggested that strain- and sex differences in the 

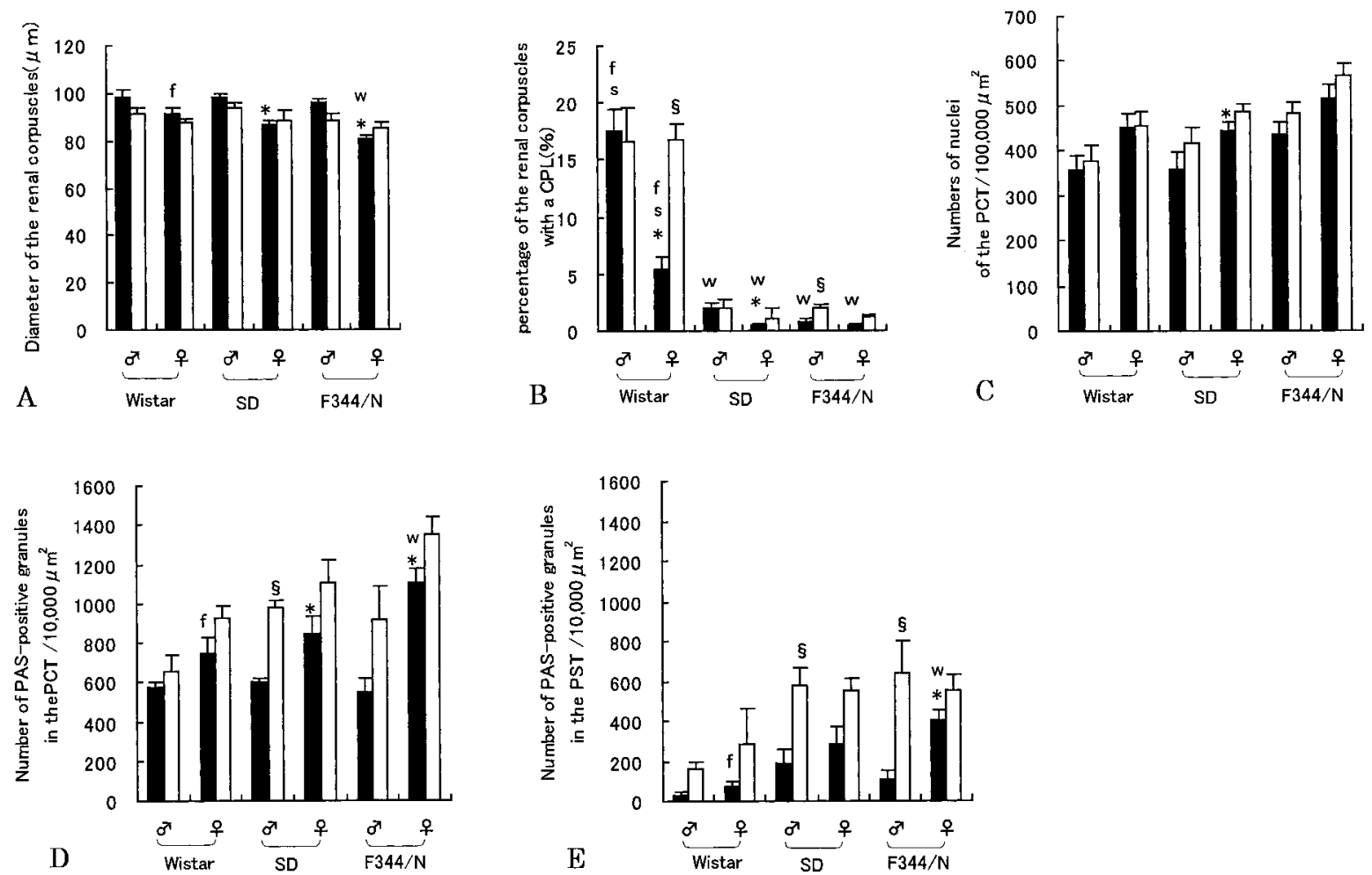

Fig. 1. Results of morphometry. A: Diameter of the renal corpuscles. B: Percentage of the renal corpuscles with a cuboidal parietal layer. C: Numbers of nuclei of the proximal convoluted tubules. D: Number of PAS-positive granules in the proximal convoluted tubular epithelium. E: Number of PAS-positive granules in the proximal straight tubular epithelium. Closed column: Intact rats. Open column: Castrated rats. Each column represents the mean \pm S.E. *: Significantly different from males of the same strain (Mann-Whiteney $\mathrm{U}$ test, $P<0.05$ ). Different letters indicate significant differences between rats of the same sex group in the three strains (ANOVA, $P<0.05$ ). w: vs Wistar, s: vs SD, f: vs F344/N. §: Significantly different from sex matched intact animals in each strain (Mann-Whiteney U test, $P<0.05$ )
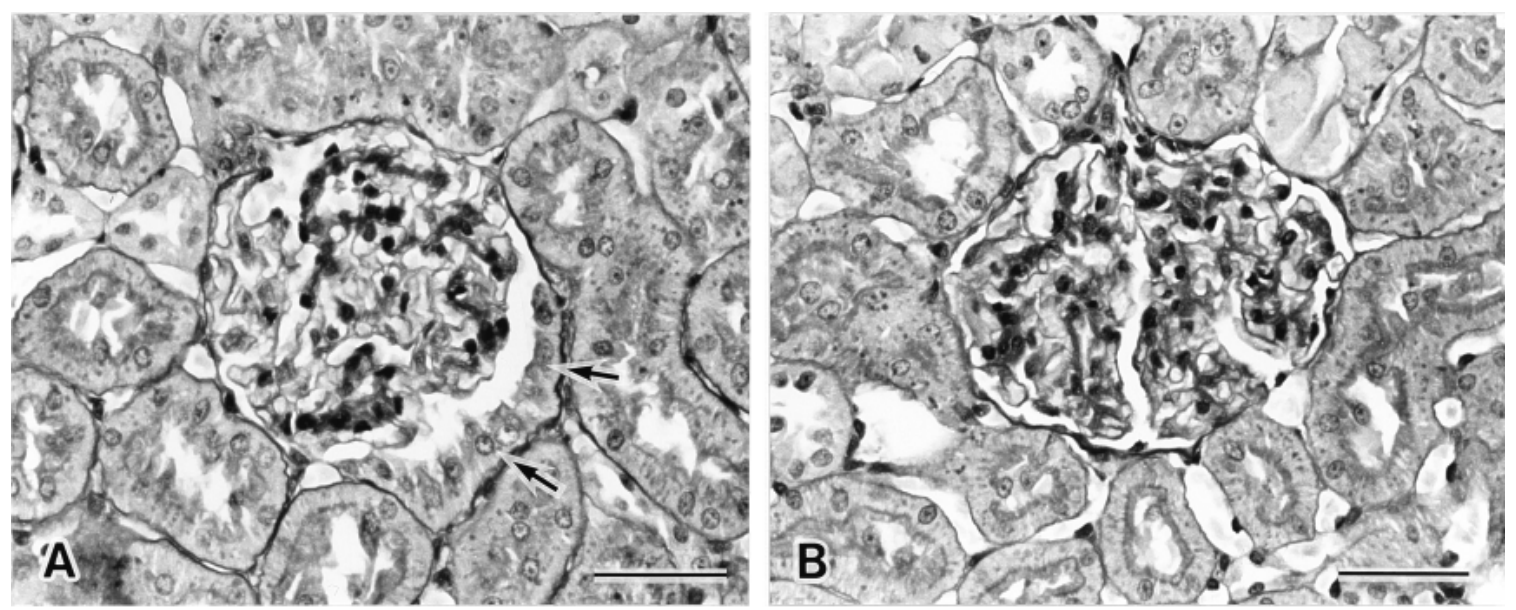

Fig. 2. Representative light micrographs of the renal corpuscles in male Wistar (A) and F344/N (B) rats. Arrows indicate the cuboidal epithelium in the parietal layer of the renal corpuscle. Renal tissues were stained with PAS. Bars: $40 \mu \mathrm{m}$.

diameter of the renal corpuscles of rat kidneys are affected by differences in the size of glomeruli rather than the height of the parietal layer of the glomerular capsule.

The CPLs in the glomerular capsule are well known mor- phological features of the male mouse kidneys [23]. In rats, it was reported that the CPL within renal corpuscles appeared in the kidneys from unilateral nephrectomized rats, rats that received a high protein diet, aging male Spra- 

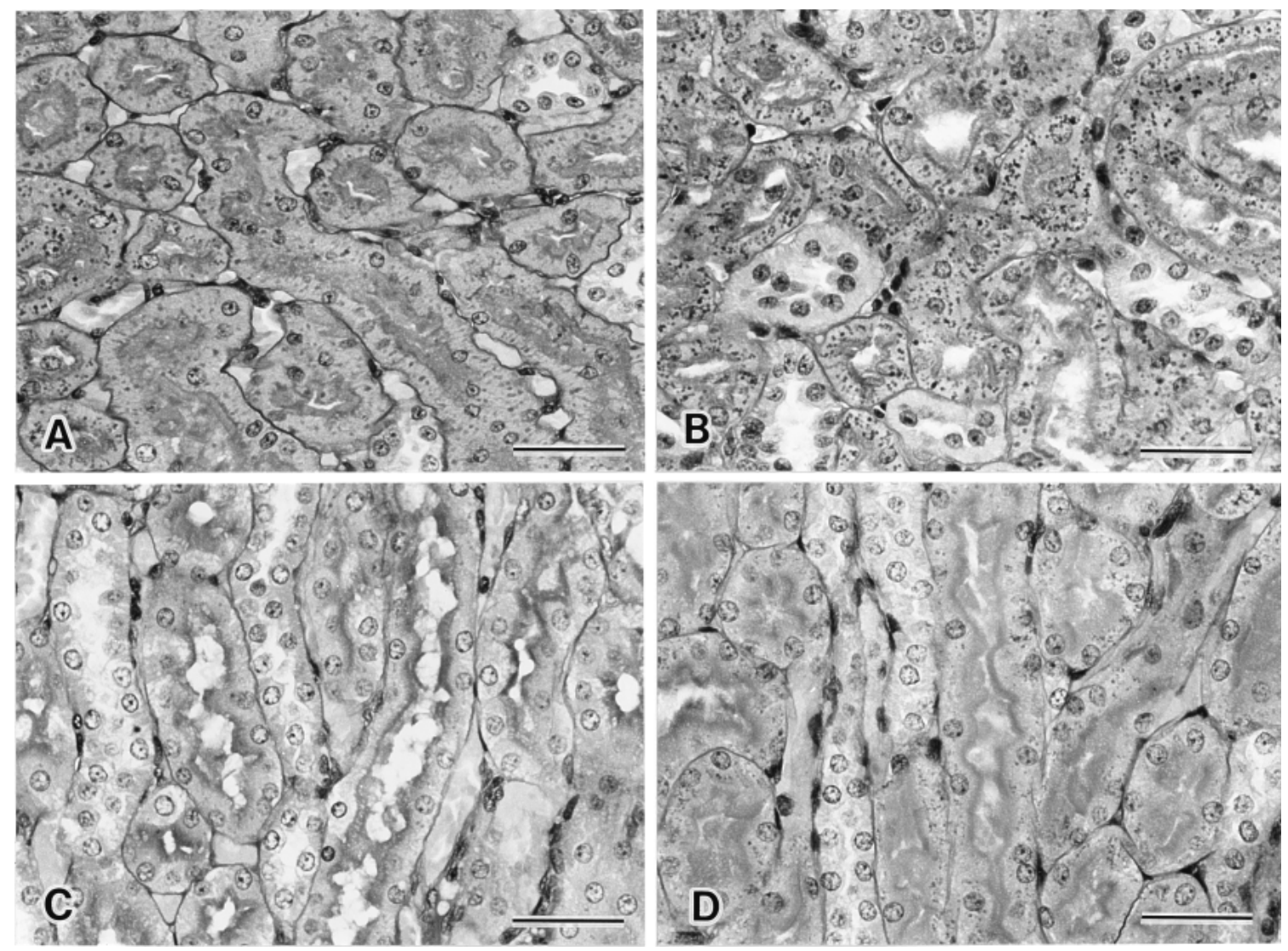

Fig. 3. Representative light micrographs of the kidneys from intact female rats. A: Cortex area in the Wistar, B: cortex area in the F344/N, C: Outer medulla area in the Wistar. D: outer medulla area in the F344/N. The PAS-positive granules are numerously observed in the proximal convoluted tubules within the cortex (A and B), rather than the proximal straight tubules within the outer medulla (C and D). The F344/N rats (B and D) contain more numerous granules than the Wistar rats (A and C) in the proximal convoluted and straight tubules. Renal tissues were stained with PAS. Bars: $40 \mu \mathrm{m}$.

gue-Dawley rats and spontaneous hypertension rats $[2,8,9$, 12]. However, the presence of the CPL in the kidneys of intact and healthy rats has not been investigated fully. Thus, the strain and sex differences of ordinary laboratory rats remain unclear. In the present study, notable findings were obtained for the percentage of the renal corpuscles with a CPL in Wistar rats. Briefly, the percentage of renal corpuscles with a CPL in the Wistar rats was significantly higher than that of other strains, and the sex difference was also remarkable in Wistar rats (males $>$ females). Haley and Bulger [9] morphologically analyzed the kidneys from three-, five- and 12-month-old male SD rats and demonstrated a significant increase of the percentage of renal corpuscles with a CPL at 12 months of age, and they hypothesized that an increase in CPL compensates for the age decrease of the absorption capabilities in the proximal tubules. We hypothesized that the absorption capabilities of proximal tubules in the Wistar rats, especially in male Wistar rats, is lower than those in the SD or F344/N rats, and the limited absorption capabilities of proximal tubules in Wistar rats are supplemented by the CPL in the renal corpuscles. However, direct or related evidence to demonstrate this hypothesis has not been reported. In the present castration experiments, an increase of the percentage of renal corpuscles with a CPL after ovariectomy in Wistar rats indicated that the development of the cuboidal cells in the parietal layer of the glomerular capsules is inhibited by estrogen.

The PCT is the longest part of the nephron, and the numbers of PCT nuclei within a given area of the cortex were counted as a parameter to infer the PCT size. The present study demonstrated no strain differences in either of the sexes, sex differences only in the SD rats, and no effects due to castration.

The PAS-positive granules were observed in the proximal tubular epithelium, and were more numerous in the PCTs than in the PSTs. Strain and sex differences were observed in both PCTs and PSTs, and the female F344/N rats showed the highest number of granules. In addition, clear effects of orchiectomy were observed in the PSTs of F344/N and SD rats and in the PCTs of SD rats. These findings indicate the inhibitory effects of androgen for the development of the PAS-positive granules in the proximal tubular epithelium in the rat kidneys. The PAS-positive granules in the proximal 
tubular epithelium were already identified as lysosomes by the electron microscopic characteristics and acid phosphatase reaction in the mouse kidneys [24-26]. Thus, we proposed that the strain and sex differences in the number of PAS-positive granules presented in the present study reflect the strain- and sex differences in the metabolic functions of rat kidneys. However, whether these granules appeared as active lysosomes digesting the molecules, which were excessively absorbed into the proximal tubular cells, or appeared as inactive residual bodies accumulating undigestible substances, was uncertain in the present study.

We proposed that the strain and sex differences presented in the present study reflect the strain and sex differences in the physiological functions of rat kidneys; for example, GFR regulation or tubular functions. Hackbarth et al. [7] analyzed the GFR and RPF of seven strains of female rats, including the sublines of Wistar and SD rats, and they demonstrated the strain differences of these two parameters. Alt et al. [1] analyzed the urinary protein of 13 strains of rats, including Wistar and SD rats, and they demonstrated that the rate of low molecular weight protein excretion was different depending on the strain and sex. However, relationships between the findings of these previous reports and those in the present study were not observed. Further studies will be required to determine the functional significances of the strain and sex differences of rat kidney morphology.

\section{REFERENCES}

1. Alt, J. M., Maess, B. and Hackbarth, H. 1985. Species and strain differences in urinary ptorein excretion. Ren. Physiol. 8: 301-309.

2. Andrews, P. M. 1981. The presence of proximal tubulelike cells in the kidney parietal epithelium in response to unilateral nephrectomy. Anat. Rec. 200: 61-65.

3. Baylis, C. 1994. Age-dependent glomerular damage in the rat. Dissociation between glomerular injury and both glomerular hypertension and hypertrophy. Male gender as a primary risk factor. J. Clin. Invest. 94: 1823-1829.

4. English, J. C., Perry, L. G., Vlaovic, M., Moyer, C. and O'Donoghue, J. L. 1994. Measurement of cell proliferation in the kidneys of Fischer 344 and Sprague-Dawley rats after gavage administration of hydroquinone. Fundam. Appl. Toxicol. 23: 397-406.

5. Gafter, U., Ben-Bassat, M. and Levi, J. 1990. Castration inhibits glomerular hypertrophy and proteinuria in uninephrectomized male rats. Eur. J. Clin. Invest. 20: 360-365.

6. Gray, J. E., Zwieten, M. J. and Hollander, C. F. 1982. Early light microscopic changes in chronic progressive nephrosis in several strains of aging laboratory rats. J. Gerontol. 37: 142150.

7. Hackbarth, H., Baunack, E. and Winn, M. 1981. Strain differences in kidney function of inbred rats: 1. Glomerular filtration rate and renal plasma flow. Lab. Anim. 15: 125-128.

8. Haensly, W. E., Granger, H. J., Morris, A. C. and Cioffe, C. 1982. Proximal-tubule-like epithelium in Bowman's capsule in spontaneously hypertensive rats. Changes with age. Am. J. Pathol. 107: 92-97.

9. Haley, D. P. and Bulger, R. E. 1983. The aging male rat: structure and function of the kidney. Am. J. Anat. 167: 1-13.
10. Hanker, J. S , Carson, K. A., Yates, P. E., Preece, J. W., Doe, D. A., Ambrose, W. W. and Coffey, J. C. Jr. 1980. Cytochemical correlates of structural sexual dimorphism in glandular tissues of the mouse. Histochemistry 68: 99-118.

11. Jedrzejewski, K. and Kugler, P. 1982. Peptidases in the kidney and urine of rats after castration. Histochemistry 74: 63-84.

12. Lalich, J. J. and Allen, J. R. 1971. Protein overload nephropathy in rats with unilateral nephrectomy. II. Ultrastructural study. Arch. Pathol. 91: 372-382.

13. Maunsbach, AB. 1966. Observations on the segmentation of the proximal tubule in the rat kidney. Comparison of results from phase contrast, fluorescence and electron microscopy. $J$. Ultrastruct. Res. 16: 239-258.

14. Mohammed, A. and Benghuzzi, H. 2000. Morphological investigation of the rat kidney after continuous administration of testosterone, dihydrotestosterone, or dehydroepiandrosterone using TCPL delivery devices. Biomed. Sci. Instrum. 36: 361366.

15. Ohtsuka, N., Sakemi, T., Tomiyoshi, Y. and Morito, F. 1997. Different effect of estrogen administration from castration on glomerular injury in unilaterally nephrectomized male Sprague-Dawley rats. Nephron 77: 445-451.

16. Oudar, O., Elger, M., Bankir, L., Ganten, D., Ganten, U. and Kriz, W. 1991. Differences in rat kidney morphology between males, females and testosterone-treated females. Ren. Physiol. Biochem. 14: 92-102.

17. Owen, R. A. and Heywood, R. 1986. Strain-related susceptibility to nephrotoxicity induced by aspirin and phenylbutazone in rats. Toxicol. Pathol. 14: 242-246.

18. Peter, C. P., Burek, J.D. and Zwieten, M. J. 1986. Spontaneous nephropathies in rats. Toxicol. Pathol. 14: 91-100.

19. Sakemi, T. and Baba, N. 1993. Castration attenuates proteinuria and glomerular injury in hyperlipidemic male Imai rats. Nephron 64: 429-435.

20. Sakemi, T., Ohtsuka, N., Tomiyosi, Y. and Morito, F. 1997. Attenuating effect of castration on glomerular injury is agedependent in unilaterally nephrectomized male Sprague-Dawley rats. Nephron 75: 342-349.

21. Schiebler, T. H. and Danner, K. G. 1978. The effect of sex hormones on the proximal tubules in the rat kidney. Cell Tissue Res. 192: 527-549.

22. Weening, J. J., Beukers, J. J., Grond, J. and Elema, J. D. 1986. Genetic factors in focal segmental glomerulosclerosis. Kidney Int. 29: 789-798.

23. Yabuki, A., Suzuki, S., Matsumoto, M. and Nishinakagawa, H. 1999. Morphometrical analysis of sex and strain differences in the mouse nephron. J. Vet. Med. Sci. 61: 891-896.

24. Yabuki, A., Suzuki, S., Matsumoto, M. and Nishinakagawa, H. 1999. Sexual dimorphism of proximal straight tubular cells in mouse kidney. Anat. Rec. 255: 316-323.

25. Yabuki, A., Suzuki, S., Matsumoto, M. and Nishinakagawa, H. 2001. Sex and strain differences in the brush border and PASpositive granules and giant bodies of the mouse renal S3 segment cells. Exp. Anim. 50: 59-66.

26. Yabuki, A., Suzuki, S., Matsumoto, M. and Nishinakagawa, H. 2003. Effects of sex hormones on the development of giant lysosomes in the proximal tubules of DBA/2Cr mouse kidney. J. Anat. 202: 445-452.

27. Zabel, M. and Schiebler, T. H. 1980. Histochemical, autoradiographic and electron microscopic investigations of the renal proximal tubule of male and female rats after castration. Histochemistry 69: 255-276. 